\title{
A PRELIMINARY STUDY OF A MHD MODEL IN THE ADAPTIVE MULTIRESOLUTION CONTEXT
}

\author{
Anna Karina F. Gomes*, Margarete O. Domingues ${ }^{\dagger}$ \\ *Pós-graduação em Computação Aplicada \\ Instituto Nacional de Pesquisas Espaciais \\ São José dos Campos, São Paulo, Brasil \\ ${ }^{\dagger}$ Laboratório Associado de Computação e Matemática Aplicada \\ Instituto Nacional de Pesquisas Espaciais \\ São José dos Campos, São Paulo, Brasil
}

Emails: annakfg@gmail.com, mo.domingues@lac.inpe.br

\begin{abstract}
On this work we present an adaptive bi-dimensional ideal magnetohydrodynamics (MHD) model using finite volume discretization, in the context of wavelet analysis. The goal of this work is to present some new results of the divergence cleaning techniques we are using concerning to the divergence of magnetic field, conservative properties of the model and computational costs.
\end{abstract}

Keywords- Fluid-dynamics, Plasma and Turbulence, Magnetohydrodynamics, Finite Volume Discretization, Compact Runge-Kutta

Resumo - Neste trabalho é apresentado um modelo magneto-hidrodinâmico (MHD) ideal bi-dimensional utilizando uma discretização por volumes finitos, no contexto da análise wavelet. O objetivo deste trabalho é apresentar novos resultados obtidos com a técnica de correção de divergência utilizada, relativos à divergência do campo magnético, propriedades conservativas do modelo e custo computacional.

Palavras-chave - Fluidodinâmica, Plasma e Turbulência, Magneto-hidrodinâmica, Discretização por Volumes Finitos, Runge-Kutta Compacto

\section{Introduction}

The interest of our research is the space plasma studies, where we have many phenomena related to magnetic fields, as the solar wind and the solar flares (Powell et al., 1999). The magnetohydrodynamics (MHD) is the study of the interaction of magnetic fields and conducting fluids, it expresses in a global view the magnetic fields influence many natural and artificial flows. In our present work, we study the numerical approach in a ideal magnetohydrodynamics model with and without divergence cleaning correction (Dedner et al., 2002) in finite volume methods with an adaptive formulation (Rousell et al., 2003; Domingues et al., 2008; Gomes, 2012; Domingues et al., 2013).

\section{Ideal MHD Model}

When the plasma components are not far from the local thermodynamic equilibrium, the plasma has a Maxwellian function of distribution, the heat flux is not important, the neutrality condition of charge holds, and the high-frequency component of magnetic field can be neglected, we have the equations of the Ideal MHD model in a conserva- tive form:

$$
\begin{aligned}
\frac{\partial \rho}{\partial t}+\nabla \cdot \rho \mathbf{u} & =0(1 \mathrm{a}) \\
\frac{\partial \rho \mathbf{u}}{\partial t}+\nabla \cdot\left(\rho \mathbf{u u}+\mathbf{I}\left(p+\frac{\mathbf{B} \cdot \mathbf{B}}{2}\right)-\mathbf{B B}\right) & =0(1 \mathrm{~b}) \\
\frac{\partial \mathbf{B}}{\partial t}+\nabla \cdot(\mathbf{u B}-\mathbf{B u}) & =0(1 \mathrm{c}) \\
\frac{\partial E}{\partial t}+\nabla \cdot\left[\left(E+p+\frac{\mathbf{B} \cdot \mathbf{B}}{2}\right) \mathbf{u}-\mathbf{B}(\mathbf{u} \cdot \mathbf{B})\right] & =0(1 \mathrm{~d})
\end{aligned}
$$

where $\mathbf{u}=\left(u_{x}, u_{y}, u_{z}\right)$ denotes the fluid velocity, $\mathbf{B}=\left(B_{x}, B_{y}, B_{z}\right)$ the magnetic induction, $\rho$ the density, $p$ the pressure, $\mathbf{I}$ the second order identity tensor and $E$ the energy given by

$$
E=\frac{p}{\gamma-1}+\rho \frac{\mathbf{u} \cdot \mathbf{u}}{2}+\frac{\mathbf{B} \cdot \mathbf{B}}{2},
$$

with $\gamma>1$ denotes the adiabatic exponent. For the magnetic field we have the divergence constraint $\nabla \cdot \mathbf{B}=0$, called magnetic flux equation. Physically, this constraint holds, but when it comes to numerical simulations some errors are propagated, leading to $\nabla \cdot \mathbf{B} \neq 0$. When the divergence constraint is not satisfied, the solution can become non-physical. Thus, to prevent this kind of behavior, we add a divergence cleaning correction to the model, as shown in the next section. It correction controls, in principle, this undesired numeric effect in the finite volume discretizations.

By adding the parabolic-hyperbolic divergence cleaning as presented in (Dedner et al., 2002), we derive two models from the Ideal MHD model: the Generalized Lagrangian Multiplier 
(GLM) and the Extended GLM (EGLM). Both formulations of Ideal MHD are two-dimensional, but only the GLM one is purely conservative. For the GLM-MHD model we have the following conservation laws equations:

$$
\begin{aligned}
& \frac{\partial \rho}{\partial t}+\nabla \cdot \rho \mathbf{u}=0(3 \mathrm{a}) \\
& \frac{\partial \rho \mathbf{u}}{\partial t}+\nabla \cdot\left(\rho \mathbf{u u}+\mathbf{I}\left(p+\frac{\mathbf{B} \cdot \mathbf{B}}{2}\right)-\mathbf{B B}\right)=0 \\
& \frac{\partial \mathbf{B}}{\partial t}+\nabla \cdot(\mathbf{u B}-\mathbf{B u}+\psi \mathbf{I})=0 \\
& \frac{\partial E}{\partial t}+\nabla \cdot\left[\left(E+p+\frac{\mathbf{B} \cdot \mathbf{B}}{2}\right) \mathbf{u}-\mathbf{B}(\mathbf{u} \cdot \mathbf{B})\right]=0(3 \mathrm{~d}) \\
& \frac{\partial \psi}{\partial t}+c_{h}^{2} \nabla \cdot \mathbf{B}=-\frac{c_{h}^{2}}{c_{p}^{2}} \psi
\end{aligned}
$$

where $\psi$ is the potential function.

For the EGLM-MHD model, we have the following equations.

$$
\begin{gathered}
\frac{\partial \rho}{\partial t}+\nabla \cdot \rho \mathbf{u}=0 \\
\frac{\partial \rho \mathbf{u}}{\partial t}+\nabla \cdot\left(\rho \mathbf{u u}+\mathbf{I}\left(p+\frac{\mathbf{B} \cdot \mathbf{B}}{2}\right)-\mathbf{B B}\right)=-\mathbf{B}(\nabla \cdot \mathbf{B}) \quad(4 \mathrm{a}) \\
\frac{\partial \mathbf{B}}{\partial t}+\nabla \cdot(\mathbf{u B}-\mathbf{B} \mathbf{u}+\psi \mathbf{I})=0 \\
\frac{\partial E}{\partial t}+\nabla \cdot\left[\left(E+p+\frac{\mathbf{B} \cdot \mathbf{B}}{2}\right) \mathbf{u}-\mathbf{B}(\mathbf{u} \cdot \mathbf{B})\right]=-\mathbf{B}(\nabla \psi) \quad(4 \mathrm{~b}) \\
\frac{\partial \psi}{\partial t}+c_{h}^{2} \nabla \cdot \mathbf{B}=-\frac{c_{h}^{2}}{c_{p}^{2}} \psi
\end{gathered}
$$

Both models presented here are formulated using the parabolic-hyperbolic correction. In addition, the parameter $c_{h}$ is evaluated as the following equation

$$
c_{h}=\frac{c_{C F L}}{\Delta t} \min (\Delta x, \Delta y),
$$

where $c_{C F L}$ is the $C F L$ number, $\Delta x, \Delta y$ are the $x$ and $y$ space steps. We also have the parameter $c_{r}=c_{p}^{2} / c_{h}$, chosen as $c_{r}=0.18$.

\subsection{Conservative Properties}

Two important conservative properties of Ideal MHD model are the energy and the time derivative of helicity. The energy should have a constant behavior over time and its equation is given by 2 . On the other hand, the time derivative of Helicity must be zero over time and its equation is

$$
\frac{\partial H}{\partial t}=-2 \int_{V}(-\mathbf{u} \times \mathbf{B}) \cdot \mathbf{B} d V,
$$

where $H$ denotes the helicity and $V$ is the volume.

\section{Numerical Discretization}

\subsection{Finite Volume}

Our ideal MHD model is hyperbolic, thus here we are using a finite volume scheme to hyperbolic conservation laws such as

$$
\frac{\partial \mathbf{U}}{\partial t}+\nabla \cdot \mathbf{F}(\mathbf{U})=\mathbf{S}(\mathbf{U})
$$

where $\mathbf{U}=\left(\rho, p, \psi, u_{x}, u_{y}, u_{z}, B_{x}, B_{y}, B_{z}\right)$ is the vector of variables and $\mathbf{F}(\mathbf{U})$ is the physical flux. To calculate $\mathbf{F}(\mathbf{U})$ of the MHD model we have to make approximations using numerical schemes, whereas $\mathbf{U}$ is not a known function.

In our work we use the Harten-Lax-van LeerDiscontinuities (HLLD) Riemann solver, as proposed in (Miyoshi and Kusano, 2005).

\section{Adaptive Multiresolution Approach}

The main idea behind MR schemes is to use the decay of wavelet coefficients of the numerical solution to obtain information on the local regularity of the solution. Small coefficients occur in regions of smoothness of the solution, where coarser grids can be used, while fine grid refinement is only applied in regions where the coefficients are significant, corresponding to strong variations [4]. Adaptive MR representations are obtained by stopping the refinement in a cell at a certain scale level, where the wavelet coefficients are non-significant. In the finite volume context, instead of using a cell-average representation on the uniform fine mesh, the MR scheme computes the numerical solution represented by cell-averages on an adaptive sparse mesh, which is formed by the cells whose wavelet coefficients are significant and above a given threshold. Since the regions of smoothness or irregularities of the solution may change with time, the MR grid at $t_{n}$ may not be convenient anymore at the next time step $t_{n+1}$. Therefore, before doing the time evolution, the representation of the solution should be interpolated onto an extended grid that is expected to be a refinement of the adaptive grid at $t_{n}$, and to contain the adaptive grid at $t_{n+1}$. Here we consider a threshold parameter $\epsilon^{\ell}$, where $l$ denotes the cell scale level, following Harten's threshold strategy.

$$
\epsilon^{\ell}=\frac{\epsilon}{|\Omega|} 2^{2(l-L+1)}, \quad 0 \leq l \leq L-1,
$$

where $L$ is the finest scale level, and $|\Omega|$ is the area of the domain. The time integration is performed by a second order Runge-Kutta scheme.

\section{Numerical Experiment}

In this section we show the comparative results obtained with MHD without correction, GLM-MHD and EGLM-MHD schemes, for different threshold parameters $\epsilon^{0}=0.05, \epsilon^{0}=0.005$ and $\epsilon^{0}=0.001$. We have tested the initial condition 2D Riemann, as shown in Table 4, with Neumann boundary condition. The other parameters chosen are $\gamma=5 / 3$, $C_{C F L}=0.3$, and $L=9$. In the adaptive context we study here, we can see that the values of divergence and time derivative of helicity are very close when calculated with GLM-MHD and EGLM-MHD models. The $\max |\nabla \cdot \mathbf{B}|$ is presented in Figure 1 over time, for $\epsilon^{0}=0.05$. We 
Table 1: 2D Riemann initial condition.

\begin{tabular}{cccc}
\hline \multicolumn{4}{c}{$x<0, y>0$} \\
\hline$\rho$ & $\rho v_{x}$ & $\rho v_{y}$ & $\rho v_{z}$ \\
1.0304 & 1.5774 & -1.0455 & -0.1016 \\
\hline$E$ & $B_{x}$ & $B_{y}$ & $B_{z}$ \\
5.7813 & 0.3501 & 0.5078 & 0.1576 \\
\hline \multicolumn{4}{c}{$x<0, y<0$} \\
\hline$\rho$ & $\rho v_{x}$ & $\rho v_{y}$ & $\rho v_{z}$ \\
1.0000 & 1.7500 & -1.0000 & 0.0000 \\
\hline$E$ & $B_{x}$ & $B_{y}$ & $B_{z}$ \\
6.0000 & 0.5642 & 0.5078 & 0.2539 \\
\hline \multicolumn{4}{c}{$x>0, y<0$} \\
\hline$\rho$ & $\rho v_{x}$ & $\rho v_{y}$ & $\rho v_{z}$ \\
1.8887 & 0.2334 & -1.7422 & 0.0733 \\
\hline$E$ & $B_{x}$ & $B_{y}$ & $B_{z}$ \\
12.999 & 0.5642 & 0.9830 & 0.4915 \\
\hline \multicolumn{4}{c}{$x>0, y>0$} \\
\hline$\rho$ & $\rho v_{x}$ & $\rho v_{y}$ & $\rho v_{z}$ \\
0.9308 & 1.4557 & -0.4633 & 0.0575 \\
\hline$E$ & $B_{x}$ & $B_{y}$ & $B_{z}$ \\
5.0838 & 0.3501 & 0.9830 & 0.3050 \\
\hline \multicolumn{4}{c}{}
\end{tabular}

can observe that it has smaller values when calculated with the corrected models GLM-MHD and EGLM-MHD. For the other values of $\epsilon^{0}$ tested the results are close.

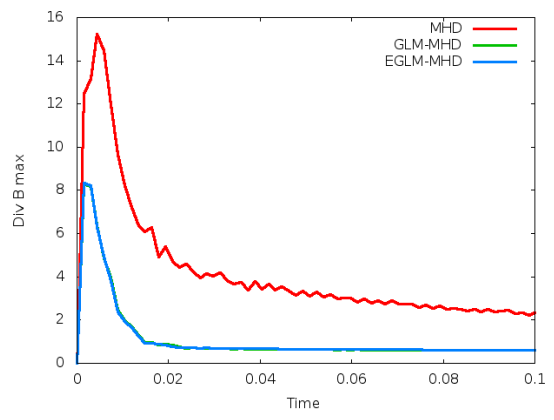

Figure 1: Maximum divergence of $\mathbf{B}$ over time for MHD, GLM and EGLM schemes for $\epsilon^{0}=0.05$ and $t=0.1$.

The energy of the MHD system was also tested and it has a constant value during all the simulations and all $\epsilon^{0}$ studied cases. The $\partial H / \partial t$ has some oscillation after $t=0.045$ for $\epsilon^{0}=0.05$, and $t=0.08$ for $\epsilon^{0}=0.001$, but these values are nearby and converge to zero machine, as expected. (a) $\epsilon^{0}=0.05$

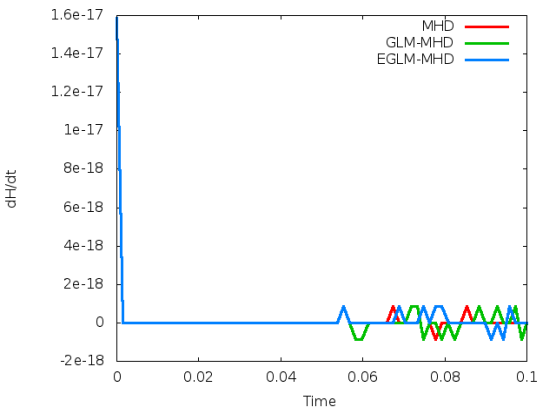

(b) $\epsilon^{0}=0.005$

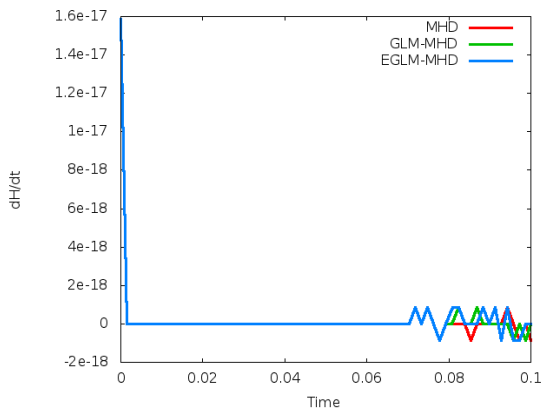

(c) $\epsilon^{0}=0.001$

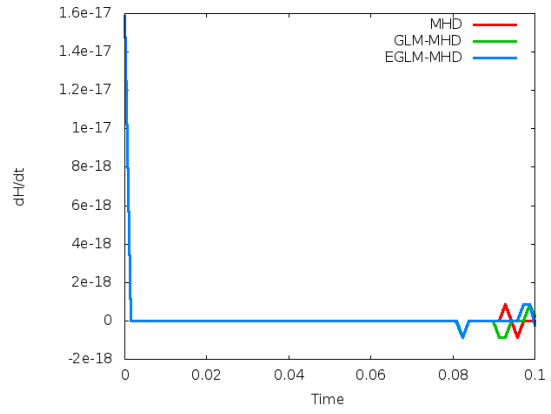

Figure 2: $\frac{\partial H}{\partial t}$ for MHD, GLM and EGLM schemes and $\epsilon^{0}=0.05, \epsilon^{0}=0.005$ and $\epsilon^{0}=0.001$, for $t=0.1$.

Table 2: CPU time and memory compression percentage of MHD models

\begin{tabular}{|c|c|c|}
\hline \multicolumn{3}{|c|}{$\epsilon^{0}=0.05$} \\
\hline & Time & Memory (\%) \\
\hline MHD & $6 \mathrm{~min} 50 \mathrm{~s}$ & 34.28 \\
\hline GLM-MHD & $6 \min 52 \mathrm{~s}$ & 34.26 \\
\hline EGLM-MHD & $6 \min 56 \mathrm{~s}$ & 34.27 \\
\hline \multicolumn{3}{|c|}{$\epsilon^{0}=0.005$} \\
\hline & Time & Memory (\%) \\
\hline MHD & $8 \min 18 \mathrm{~s}$ & 41.19 \\
\hline GLM-MHD & $8 \min 20 \mathrm{~s}$ & 41.25 \\
\hline EGLM-MHD & $8 \min 28 \mathrm{~s}$ & 41.26 \\
\hline \multicolumn{3}{|c|}{$\epsilon^{0}=0.001$} \\
\hline & Time & Memory (\%) \\
\hline MHD & $9 \mathrm{~min} 37 \mathrm{~s}$ & 47.56 \\
\hline GLM-MHD & $9 \min 47 \mathrm{~s}$ & 47.76 \\
\hline EGLM-MHD & $9 \min 49 \mathrm{~s}$ & 47.77 \\
\hline
\end{tabular}

The MHD and GLM-MHD models have similar maximum divergence values over time. How- 
ever, in Figure 3 we can see that the solution obtained with the MHD model without correction has undesired structures. In Table 2, we observe that the CPU time of all MHD models tested here are very close, what enforce the simulate the GLM-MHD model is not so expensive. The space visualization of absolute magnetic field

(a) MHD

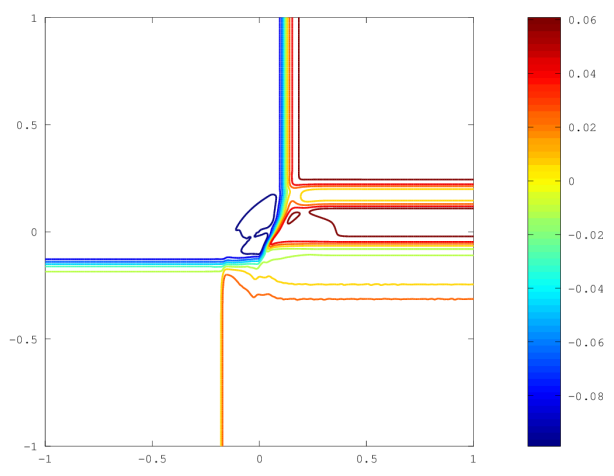

(b) GLM-MHD

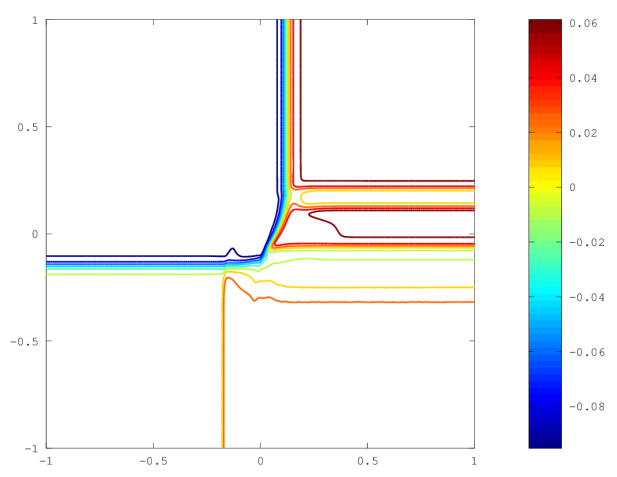

Figure 3: z-component of velocity obtained with MHD and GLM-MHD models for $\epsilon^{0}=0.05, t=$ 0.1 and $L=9$.

divergence in Figures 4 (b), (c) shows that we have larger values of $\nabla \cdot \mathbf{B}$ when the solution of MHD model presents more structures like shocks. Therefore, the adaptive mesh for the final time, in Figure 4 (a), presents more refinement in the regions where the variables have more structures ( $29.93 \%$ of the cells). In this case, the adaptive mesh is obtained as the union of all the individual adaptive meshes. Also, it is important to notice that the divergence values for the GLM-MHD scheme are bounded in the interval $[0,0.8]$, and for the MHD scheme these values are bounded in the interval [0,2.3]. (a) Adaptive mesh

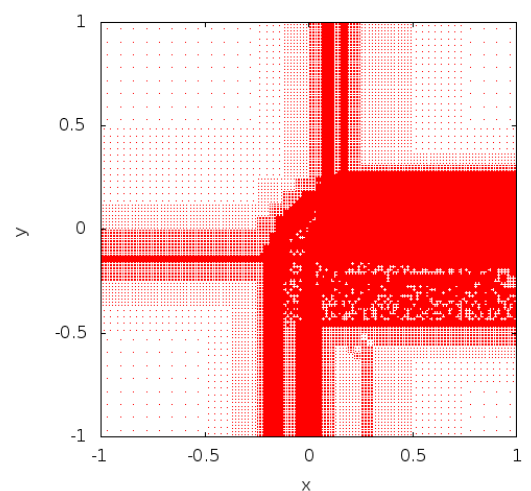

(b) GLM-MHD

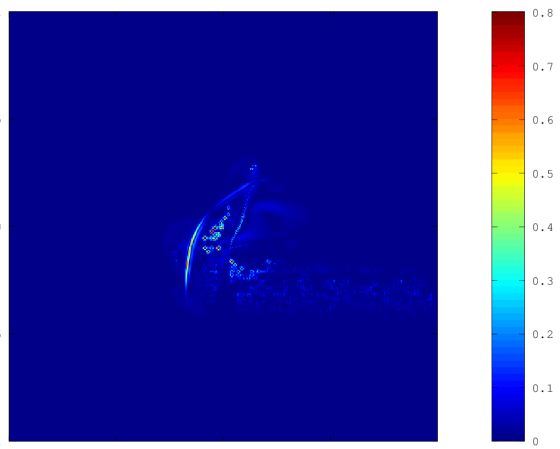

(c) MHD

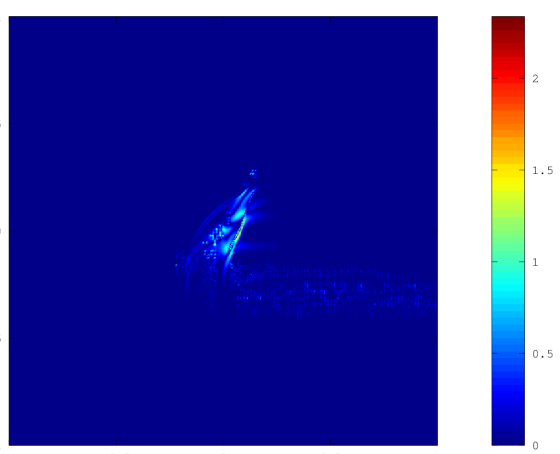

Figure 4: Adaptive mesh and $\nabla \cdot \mathbf{B}$ obtained with MHD without correction and GLM-MHD model for $\epsilon^{0}=0.05$ and $t=0.1$.

\section{Conclusions}

The results present in this study are encouraging to this wavelet adaptive method. First, we obtained that the values of magnetic field divergence and conservative proprieties are very close, when calculated with GLM-MHD and EGLMMHD models in the adaptive simulations. Moreover, they are closer to the FV scheme method ones. The $\max |\nabla \cdot \mathbf{B}|$ over time has smaller values when calculated with the corrected models GLM and EGLM and non undesired structures are presented. Finally, we observe in this study that the CPU time of all tested MHD adaptive 
models are very close, however the GLM-MHD model is slightly computationally cheaper, and as it has theoretical conservation propriety, it is our choice for further studies .

\section{Acknowledgements}

The authors thankfully acknowledge financial support from CAPES (grants 86/2010-29), CNPq (grants 141741/2013-9, 312479/2012-3, 551006/2011-0, 483226/2011-4 , 306828/2010-3, $307511 / 2010-3,486165 / 2006-0)$ and FAPESP (grants 2012/06577-5, 2012/072812-2, 2007/07723-7). We are grateful to Varlei E. Menconi (FAPESP grants 2008/09736-1 and PCI-INPE) for their helpful computational assistance.

\section{References}

Dedner, A., Kemm, F., Kröner, D., Munz, C.D., T.Schnitzer and Wesenberg, M. (2002). Hyperbolic divergence cleaning for the MHD equations, Journal of Computational Physics 175: 645-673. DOI: 10.1006/jcph.2001.6961

Domingues, M. O., Gomes, A. K. F., Gomes, S., Mendes, O., Pierro, B. D. and Schneider, K. (2013). Extended generalized lagrangian multipliers for magnetohydrodynamics using adaptive multiresolution methods, ESAIM Proceedings (to appear).

Domingues, M. O., Gomes, S. M., Roussel, O. and Schneider, K. (2008). An adaptative multiresolution scheme with local time stepping for evolutionary PDEs, Journal of Computational Physics 227: 3758-3780. DOI: 10.1016/j.jcp.2007.11.046

Gomes, A. K. F. (2012). Análise multirresolução adaptativa no contexto da resolução numérica de um modelo de magnetohidrodinâmica ideal, Master's thesis, Instituto Nacional de Pesquisas Espaciais (INPE), São José dos Campos.

Miyoshi, T. and Kusano, K. (2005). A multi-state HLL approximate riemann solver for ideal magnetohydrodynamics, Journal of Computational Physics 208: 315-344. DOI:

10.1016/j.jcp.2005.02.017

Powell, K. G., Roe, P. L., Linde, T. J., Gombosi, T. I. and Zeeuw, D. L. D. (1999). A solutionadaptive upwind scheme for ideal magnetohydrodynamics, Journal of Computational Physics 154: 284-309. DOI: 10.1006/jcph.1999.6299

Rousell, O., Schneider, K., Tsigulin, A. and Bockhorn, H. (2003). A conservative fully adaptive multiresolution algorithm for parabolic PDEs, Journal of Computational Physics 188: 493-523. DOI: 10.1016/S0021-9991(03)00189-X 